\title{
Trypanosoma cruzi: Influence of predominant bacteria from indigenous digestive microbiota on experimental infection in mice
}

\author{
R. Duarte ${ }^{\text {a }}$, A.M. Silva ${ }^{\text {a }}$, L.Q. Vieira ${ }^{\text {b }}$, L.C.C. Afonso ${ }^{\text {c }}$, J.R. Nicoli ${ }^{\text {a,* }}$ \\ a Departamento de Microbiologia, ICB, Universidade Federal de Minas Gerais, Belo Horizonte, MG, Brazil \\ ${ }^{\mathrm{b}}$ Departamento de Bioquímica-Imunologia, ICB, Universidade Federal de Minas Gerais, Belo Horizonte, MG, Brazil \\ ${ }^{\mathrm{c}}$ Departamento de Ciências Biológicas, ICEB/NUPEB, Universidade Federal de Ouro Preto, Ouro Preto, MG, Brazil
}

Received 4 March 2005; received in revised form 2 June 2005; accepted 7 June 2005

Available online 20 July 2005

\begin{abstract}
To verify the influence of some predominant components from indigenous microbiota on systemic immunological responses during experimental Chagas disease, germ-free NIH Swiss mice were mono-associated with Escherichia coli, Enterococcus faecalis, Bacteroides vulgatus or Peptostreptococcus sp. and then infected with the Y strain of Trypanosoma cruzi. All the mono-associations predominantly induced a Th1 type of specific immune response to the infection by $T$. cruzi. A direct correlation was observed between a higher survival rate and increased IFN- $\gamma$ and TNF- $\alpha$ production $(P<0.05)$ in E. faecalis-, B. vulgatus-, and Peptostreptococcus-associated mice. Moreover, higher levels of anti-T. cruzi IgG1 and anti-T. cruzi IgG2a were also found in mono-associated animals after infection. On the other hand, with the exception of E. faecalis-associated mice, mono-association induced a lower IL-10 production after infection $(P<0.05)$ when compared with germ-free animals. Interestingly, spleen cell cultures from non-infected germ-free and mono-associated mice spontaneously produced higher levels $(P<0.05)$ of IL-10 than cultures from infected monoassociated mice, except again for E. faecalis-associated animals. In conclusion, the presence of the components of the indigenous microbiota skews the immune response towards production of inflammatory cytokines during experimental infection with $T$. cruzi in gnotobiotic mice. However, the degree of increase in production of cytokines depends on each bacterial component.

(c) 2005 Elsevier Inc. All rights reserved.
\end{abstract}

Keywords: Microbiota; Trypanosoma cruzi; Cytokines; Immunoglobulins; Gnotobiotic mice

\section{Introduction}

The human gastrointestinal tract harbors one of the most complex ecosystems known in microbial ecology with bacterial populations reaching $10^{10}-10^{11}$ viable cells/g of contents in its lower portions. These organisms may belong to about 400 different bacterial species, although it is believed that only $20-40$ of them reach predominant levels, consisting of $99 \%$ of the total community (Berg, 1996). Concerning the human predominant fecal bacteria, two population levels can be

\footnotetext{
${ }^{*}$ Corresponding author. Fax: +55 3134992730.

E-mail address: jnicoli@icb.ufmg.br (J.R. Nicoli).
}

distinguished: (i) the dominant microbiota $\left(10^{9}-\right.$ $10^{11}$ cells/g of contents) constituted only by obligate anaerobes (Bacteroides, Bifidobacterium, Eubacterium, Peptostreptococcus, and Fusobacterium) and (ii) the sub-dominant microbiota $\left(10^{7}-10^{8}\right.$ cells/g of contents) containing predominantly facultative anaerobic and microaerophilic bacteria (Escherichia coli, Enterococcus, and Lactobacillus). In healthy hosts, the presence of this microbiota has a very large impact on various aspects of function and metabolism such as metabolic rate, gastrointestinal function, specific and quantitative aspects of immune function, and the many aspects of biochemical homeostasis. Only the predominant species have population levels high enough to be considered 
as responsible for the three main functions of the intestinal microbiota which have considerable importance for the host health: (i) the colonization resistance, (ii) the immunomodulation, and (iii) the nutritional contribution for the host (MacFarland, 2000). Presently, available data also indicate that this indigenous microbiota almost always has a profound influence on the host-parasite relationship. As an example, it is well known that the presence of intestinal microbiota is essential for the pathogenicity of some protozoa and helminthes such as Entamoeba histolytica (Phillips and Wolfe, 1959), Nippostrongylus brasiliensis (Wescott and Todd, 1964), Nematospiroides dubius (Wescott, 1968), Trichinella spiralis (Przyjalkowski and Wescott, 1969), Eimeria tenella (Visco and Barnes, 1972), Ascaridia galli (Johnson and Reid, 1973), Trichuris suis (Rutter and Beer, 1975), Eimeria falciformis (Owen, 1975), Eimeria ovinoidalis (Gouet et al., 1984), and Giardia duodenalis (Torres et al., 2000). In contrast, this microbiota can reduce the pathological consequences of other infectious diseases as described for experimental infections with Trypanosoma cruzi (Silva et al., 1987), Cryptococcus neoformans (Salkowski et al., 1987), Strongyloides venezuelensis (Martins et al., 2000), and almost all enteropathogenic bacteria (Clostridium difficile, Clostridium perfringens, E. coli, Pseudomonas aeruginosa, Salmonella typhimurium, Shigella flexneri, and Vibrio cholerae) (Wilson, 1995). Experimental infections with Raillietina cesticillus (Reid and Botero, 1967) and Isopora suis (Harleman and Meyer, 1984) are two of the very few cases, where the normal microbiota has no influence on the course of a disease.

Trypanosoma cruzi is the causative agent of Chagas disease in man and determines a systemic infection that is controlled, although not completely eliminated, by $\mathrm{T}$ cell-dependent immune responses. Control of parasitemia in the acute phase of infection is critically dependent on intracellular killing by cytokine-activated macrophages. In this way, different studies indicate the crucial role of IFN- $\gamma$, IL-12 (Michailowsky et al., 2001), and TNF- $\alpha$ (Silva et al., 1995) as well as NO (Vespa et al., 1994) in host resistance to infection with T. cruzi.

As cited above, infection with the intracellular parasite $T$. cruzi is more severe in germ-free animals, as shown by a higher mortality when compared with conventional controls (Silva et al., 1987). Germ-free mice also displayed earlier and higher parasitemia than conventional controls. Moreover, tissues from germ-free mice were more intensively parasitized and presented a more aggressive inflammatory response. Germ-free mice infected with $T$. cruzi presented a stronger local reaction to subcutaneous injection of formalin-killed parasites as determined by footpad swelling than conventional animals (Furarah et al., 1991). Recent data showed higher IFN- $\gamma, \mathrm{TNF}-\alpha$, and NO production by spleen cell cultures, and higher blood levels of IgG1 in conventional mice infected with $\mathrm{Y}$ strain of $T$. cruzi when compared to their germ-free counterparts (Duarte et al., 2004). However, these data concern the whole microbiota and there is no information about the role of individual components of the indigenous bacterial ecosystem on these differences.

To investigate the role of individual components of the indigenous microbiota on the immune response to a systemic parasitic infection, germ-free mice were monoassociated with Gram positive (Peptostreptococcus sp.) and Gram negative (Bacteroides vulgatus) obligate anaerobic bacteria or Gram positive (Enterococcus faecalis) and Gram negative (E. coli) facultative bacteria, pertaining to the predominant human fecal microbiota, and subsequently infected with $T$. cruzi. Survival rate, cytokine, and NO productions by spleen cell cultures, and $\mathrm{IgG} 1$ and $\mathrm{IgG} 2 \mathrm{a}$ serum concentrations were determined.

\section{Materials and methods}

\subsection{Mice}

Germ-free 21-day-old NIH mice of both sexes were used in this study. The matrices were obtained from Taconic Farms (Germantown, NY, USA) and maintained in the germ-free facility of the Instituto de Ciências Biológicas (Universidade Federal de Minas Gerais, Belo Horizonte, MG, Brazil) for several generations. Germ-free animals were housed in flexible plastic isolators (Standard Safety, McHenry, IL, USA) and handled according to established procedures. Experiments with gnotobiotic mice were carried out in micro-isolators (UNO Roestvastaal B.V., Zevenar, The Netherlands). Water and commercial autoclavable diet (Nuvital, Curitiba, PR, Brazil) were steam sterilized and administered ad libitum. Microbiological status of germ-free and gnotobiotic mice was performed by routine culture of recently collected feces in brain heart infusion (BHI) broth medium (Oxoid, Hampshire, England) and fluid thioglycollate medium (Difco, Detroit, MI, USA). Fecal cultures were incubated at 37 and $25^{\circ} \mathrm{C}$ during $72 \mathrm{~h}$. Controlled lighting ( $12 \mathrm{~h}$ light, $12 \mathrm{~h}$ dark) was used for all the animals. All the animals received humane care as outlined in the "Guide for the Care and Use of Laboratory Animals" of the National Research Council (1996).

\subsection{Bacteria}

Escherichia coli, Enterococcus faecalis, Bacteroides vulgatus, and Peptostreptococcus sp. were all isolated from feces of healthy human volunteers. The bacteria were maintained at $-86^{\circ} \mathrm{C}$ in $\mathrm{BHI}$ broth medium (Oxoid) supplemented with glycerol $10 \%$. Identity of 
these bacteria was regularly confirmed by using API identification kits (BioMérieux, Marcy l'Etoile, France). Inoculum of each bacterium was prepared from cultured BHI broth medium (Oxoid) incubated at $37^{\circ} \mathrm{C}$ under aerobic conditions for E. coli and E. faecalis or in an anaerobic chamber (Forma Scientific, Marietta, $\mathrm{OH}$, USA, containing an atmosphere of $\mathrm{N}_{2} 85 \%, \mathrm{H}_{2} 10 \%$, and $\mathrm{CO}_{2} 5 \%$ ) for B. vulgatus and Peptostreptococcus sp. Mono-association was carried out by the administration of a single dose $\left(0.2 \mathrm{ml}\right.$ containing about $10^{8}$ viable cells $)$ of each bacterial strain to germ-free mice by intra-gastric intubation 10 days before the experimental challenge with $T$. cruzi. As controls, germ-free mice were inoculated intra-gastrically with $0.2 \mathrm{ml}$ of sterile saline 10 days before challenge.

\subsection{Bacterial counts in feces from mono-associated mice}

Feces freshly collected from each gnotobiotic group were diluted 100-fold in saline and vortexed. Serial 10fold dilutions were made and $0.1 \mathrm{ml}$ plated onto BHI agar medium (Oxoid). Plates were incubated at $37^{\circ} \mathrm{C}$ for $24-48 \mathrm{~h}$ for bacterial counts (under aerobic or anaerobic conditions depending on the bacteria).

\subsection{Parasite}

Trypanosoma cruzi, Y strain (Laboratório de Parasitologia e Histopatologia, DECBI-ICEB, Universidade Federal de Ouro Preto, Ouro Preto, MG, Brazil) was used and maintained by weekly successive transfers in $\mathrm{NIH}$ conventional mice.

\subsection{Experimental infection}

Germ-free and mono-associated mice were inoculated with $5 \times 10^{3}$ blood trypomastigotes obtained from mice in the acute phase of the infection. Briefly, blood was collected from the axillary plexus in 3.8\% sodium citrate/PBS and parasitemia evaluated according to Brener (1962). The number of parasites was adjusted to the desired inocula in PBS. All the manipulations were performed in a laminar flow hood. A sample was seeded in fluid thioglycollate medium (Difco) and BHI broth medium (Oxoid) for control of asepsis.

\subsection{Experimental design}

Five groups of mice were used to obtain the following microbiological status: (i) germ-free control, (ii) E. coli mono-associated, (iii) E. faecalis mono-associated, (iv) B. vulgatus mono-associated, and (v) Peptostreptococcus sp. mono-associated. Several of these germ-free and gnotobiotic groups (5-10 mice for each group according to the experiments) were used separately for determina- tions of survival rate, cytokine levels, and NO and immunoglobulin concentrations. Survival rate was determined over 40 days and accumulated mortality noted. For immunological determinations, groups of mice were killed just before or seven days after the $T$. cruzi inoculation. Three repetitions were done for each determination.

\subsection{Trypanosoma cruzi antigens}

Antigens from T. cruzi were obtained from trypomastigotes cultured on VERO cells. Parasites were harvested from the supernatant, centrifuged at $3000 \mathrm{~g}$ during $15 \mathrm{~min}$ at $4{ }^{\circ} \mathrm{C}$, and washed three times in PBS. The number of trypomastigotes was adjusted to $10^{8} \mathrm{cells} / \mathrm{ml}$, and submitted to five cycles of freezing and thawing. The parasite extract was homogenized, aliquoted, and maintained at $-20^{\circ} \mathrm{C}$ until use.

\subsection{Cytokine quantitation in spleen cultures}

Mice from gnotobiotic groups were killed and the spleen was removed, macerated aseptically in RPMI1640 (Sigma Chemical, St. Louis, MO, USA), and centrifuged at $1000 \mathrm{~g}$ for $10 \mathrm{~min}$. Erythrocytes were lysed, and spleen cells were washed and centrifuged twice. Then, cells were suspended in RPMI-1640 supplemented with $10 \%$ fetal bovine serum, $50 \mu \mathrm{M} \beta$-mercaptoethanol (Sigma), $10 \mathrm{mg} / \mathrm{ml}$ gentamicin sulfate, and $3.2 \mathrm{mM}$ L-glutamine (Sigma). Cell number and viability were assessed by trypan blue dye exclusion on a Neubauer hematocytometer and the final cell suspension was adjusted to $5 \times 10^{6}$ cells $/ \mathrm{ml}$. Cells were cultured in $24-w e l l$ tissue culture plates in the absence or presence of the $T$. cruzi antigen $(50 \mu l$ of the antigen preparation $/ \mathrm{ml}$ of culture, see above). Triplicate cultures of all experiments, using three animals for each one, were incubated for $72 \mathrm{~h}$ at $37^{\circ} \mathrm{C}$ in $5 \% \mathrm{CO}_{2}$. After incubation, supernatants were harvested and stored at $-86^{\circ} \mathrm{C}$ for cytokine assay. Duoset kits for mouse IFN- $\gamma$, TNF- $\alpha$, and IL-10 (R\&D Systems, Minneapolis, MN, USA) were used to determine cytokine levels in culture supernatants according to the manufacturer's instructions. Absorbance was read at $450 \mathrm{~nm}$ on a microplate reader (Bio-Rad Laboratories, Hercules, CA, USA). The sensitivities of the assays were 72,62 , and $36 \mathrm{pg} / \mathrm{ml}$ for IFN- $\gamma$, TNF- $\alpha$, and IL-10, respectively.

\subsection{Detection of NO production in spleen cultures}

NO production was determined according to Green et al. (1982). The nitrite content in the supernatants was measured by adding $50 \mu 1$ of Griess reagent to $50 \mu 1$ of the sample in 96-well plates, reading the optical density (OD) at $550 \mathrm{~nm} 15 \mathrm{~min}$ later and comparing with the OD curves of serial dilutions of sodium nitrite in complete medium. 


\subsection{Immunoglobulin analysis in serum}

Total and parasite-specific IgG1 and IgG2a levels in serum were evaluated by capture ELISA (dilution 200fold). To detect IgG1 and IgG2a isotypes, biotinylated rat antibodies anti-mouse IgG1 and IgG2a (Southern Biotechnology Associates, Birmingham, AL, USA) were used. Absorbance at $492 \mathrm{~nm}$ was determined with an ELISA plate reader (Bio-Rad). The concentrations of each immunoglobulin were determined using the respective purified mouse standard (Southern Biotechnology Associates).

\subsection{Statistical analysis}

The results shown are from one representative of at least three independently performed. Statistical significance of the results was evaluated by analysis of variance (ANOVA) for all data, except survival, for which LogRank test was used. The level of significance was set at $P<0.05$.

\section{Results}

\subsection{Survival rate}

All bacteria established in the digestive tract of gnotobiotic mice. Fecal counts yielded $10^{9}$ colony-forming units/g of feces within two days, and these high levels were maintained until the end of the experiment. Fig. 1 shows experiments on survival of germ-free and gnotobiotic mice challenged with T. cruzi, Y strain. A significantly higher survival rate was observed for gnotobiotic animals mono-associated with $E$. faecalis $(P=0.034), B$. vulgatus $(P=0.035)$ or Peptostreptococcus $(P=0.029)$ when compared to control group. Mice mono-associated with $E$. coli survived slightly longer than control animals but the results were not statistically significant $(P=0.103)$.

\subsection{Cytokine determinations}

To determine if mono-association with bacteria would alter the response of germ-free mice to $T$. cruzi antigens, spleen cells from germ-free and mono-associated mice were cultured in the presence of $T$. cruzi homogenate. As seen in Fig. 2A, except for the E. coli mono-associated group, in vitro addition of $T$. cruzi homogenate triggered IFN- $\gamma$ production. Moreover, Fig. 2A shows similar basal levels of IFN- $\gamma$ in the supernatants of spleen cultures from germ-free and gnotobiotic NIH mice, before the infection, except for the Peptostreptococcus-associated group $(P<0.05)$. When spleen cells were cultured with $T$. cruzi antigens during $72 \mathrm{~h}$, a significant increase $(P<0.05)$ in IFN- $\gamma$ production

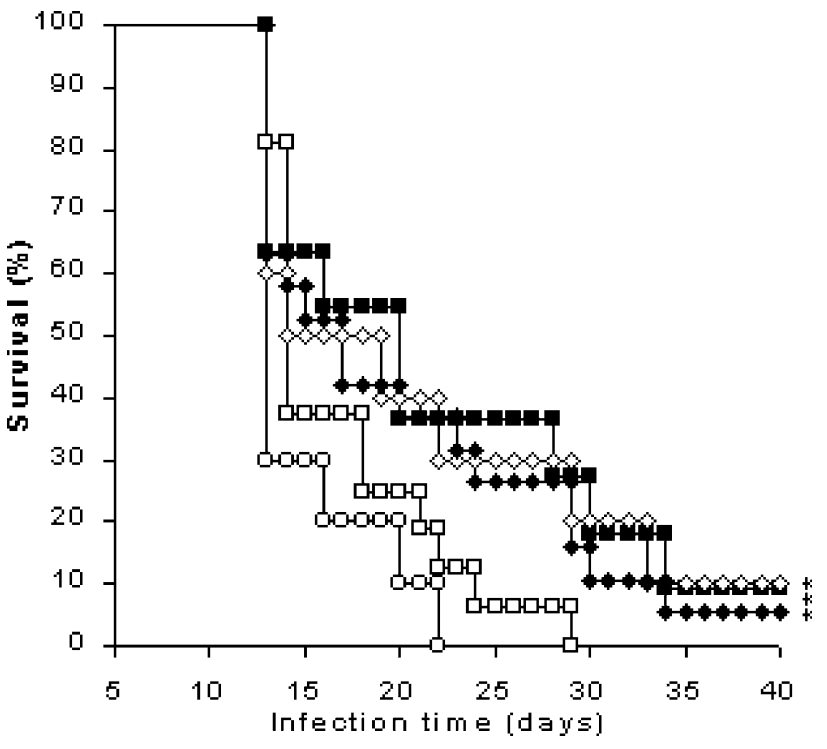

Fig. 1. Survival of Swiss/NIH mice, germ-free $(\bigcirc)$ or mono-associated with Escherichia coli $(\square)$, Enterococcus faecalis $(\mathbf{\square})$, Bacteroides vulgatus $(\diamond)$, and Peptostreptococcus sp. $(\diamond)$ after intraperitoneal challenge with $5 \times 10^{3}$ trypomastigotes of Trypanosoma cruzi. Data of one representative experiment of three performed independently (10 mice/experiment) are shown. $(*)$, Statistically significant difference as evaluated by Log Rank test $(P<0.05)$ of mono-associated groups compared to the germ-free group.

was observed for germ-free animals but very different responses were obtained for the different bacterial associations. These responses to $T$. cruzi antigens ranged from a small increase observed for $E$. coli-associated mice to the production of high levels of IFN- $\gamma$ by spleen cell from cultures of Peptostreptococcus-associated animals $(P<0.05)$. Seven days after intraperitoneal infection with $T$. cruzi (Fig. 2B), significantly higher IFN- $\gamma$ levels $(P<0.05)$ were found in spleen cell culture supernatants from gnotobiotic mice stimulated with parasite antigens $(P<0.05)$. In germ-free animals, this increase in IFN- $\gamma$ production after experimental infection was not observed, even in supernatants of spleen cells cultured in the presence of $T$. cruzi antigens.

Before experimental infection, basal TNF- $\alpha$ production was similar in germ-free and gnotobiotic animals (Fig. 3A). Increased levels of this cytokine were observed in germ-free and gnotobiotic mice when spleen cells were stimulated with parasite antigens, and higher values were observed for E. coli, E. faecalis, and Peptostreptococcusassociated groups $(P<0.05)$. After infection (Fig. 3B), again, higher TNF- $\alpha$ levels were found in supernatants of spleen cell cultures from the gnotobiotic mice stimulated with $T$. cruzi antigens when compared to their germ-free and B. vulgatus-associated counterparts $(P<0.05)$, particularly for Peptostreptococcus-associated group.

Similar NO levels were observed in supernatants of spleen cultures of non-infected germ-free and gnotobiotic animals (Table 1). No increase was found when antigens were added to the culture (data not shown). Seven 

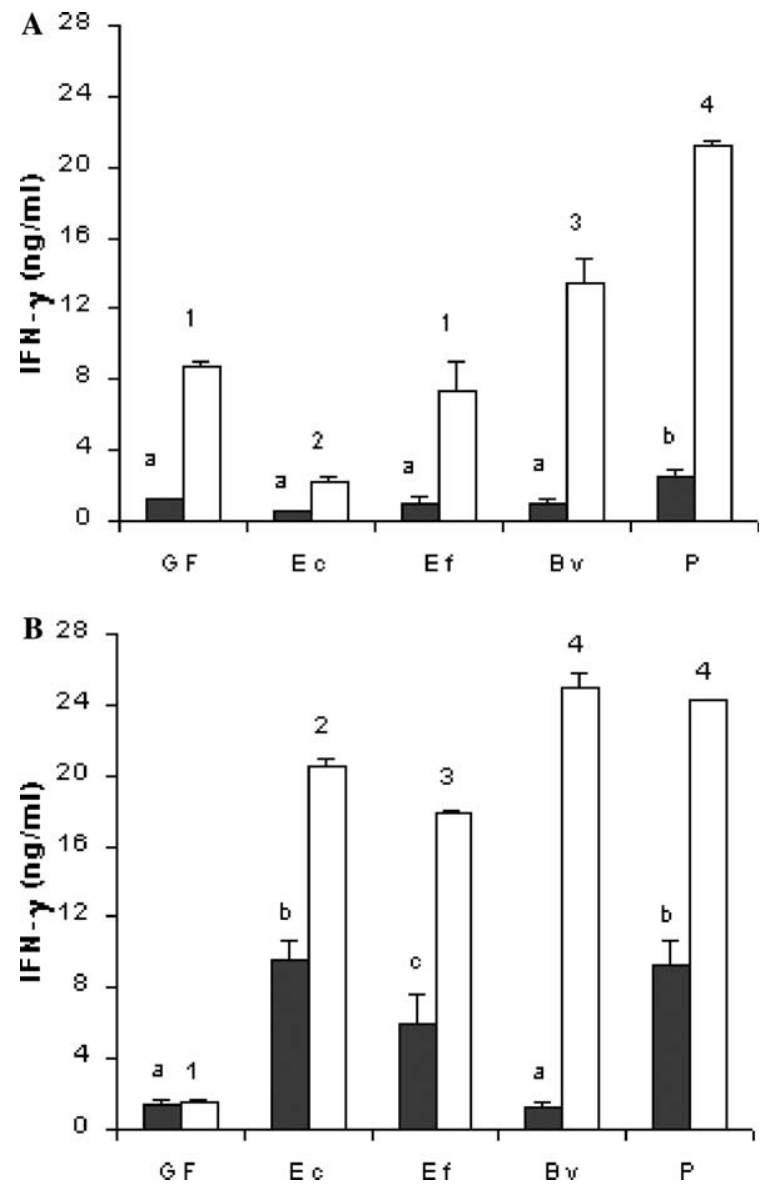

Fig. 2. IFN- $\gamma$ production by spleen cells from Swiss/NIH mice, germfree (GF) or mono-associated with Escherichia coli (EC), Enterococcus faecalis (EF), Bacteroides vulgatus (BV), and Peptostreptococcus sp. (P) before (A) and seven days after (B) intraperitoneal challenge with $5 \times 10^{3}$ trypomastigotes of Trypanosoma cruzi. Cells were cultured for $72 \mathrm{~h}$ in the absence ( $\square$ ) or presence ( $\square$ ) of T. cruzi antigen. Each bar represents the mean of one representative experiment of three performed (three mice/experiment; spleens from each animal were cultured individually in each experiment). Vertical lines represent standard deviations of the means. ${ }^{\mathrm{a}, \mathrm{b}, \mathrm{c}}$ Different letters and ${ }^{1,2,3,4}$ different numbers between non-stimulated and stimulated groups, respectively, as evaluated by analysis of variance $(P<0.05)$.

days after infection, an increase in NO production was observed only in spleen cell culture from $E$. coli- and Peptostreptococcus-associated mice $(P<0.05)$ (Table 1).

Before infection, background production of IL-10 was higher in supernatants of spleen cell cultures of germ-free and E. faecalis-associated mice than in the other groups (Fig. 4A). Furthermore, the presence of $T$. cruzi antigen in culture induced an increased IL-10 production, proportionally higher in the E. coli-associated animals. After infection (Fig. 4B), the same significant difference $(P<0.05)$ between germ-free and E. faecalisassociated mice and the other groups was observed, but only when cells were stimulated with $T$. cruzi antigens. Table 2 shows the impact of infection on the cytokine balance. After infection, the highest IFN- $\gamma / \mathrm{IL}-10$ ratio was found in the $E$. coli mono-associated group. This
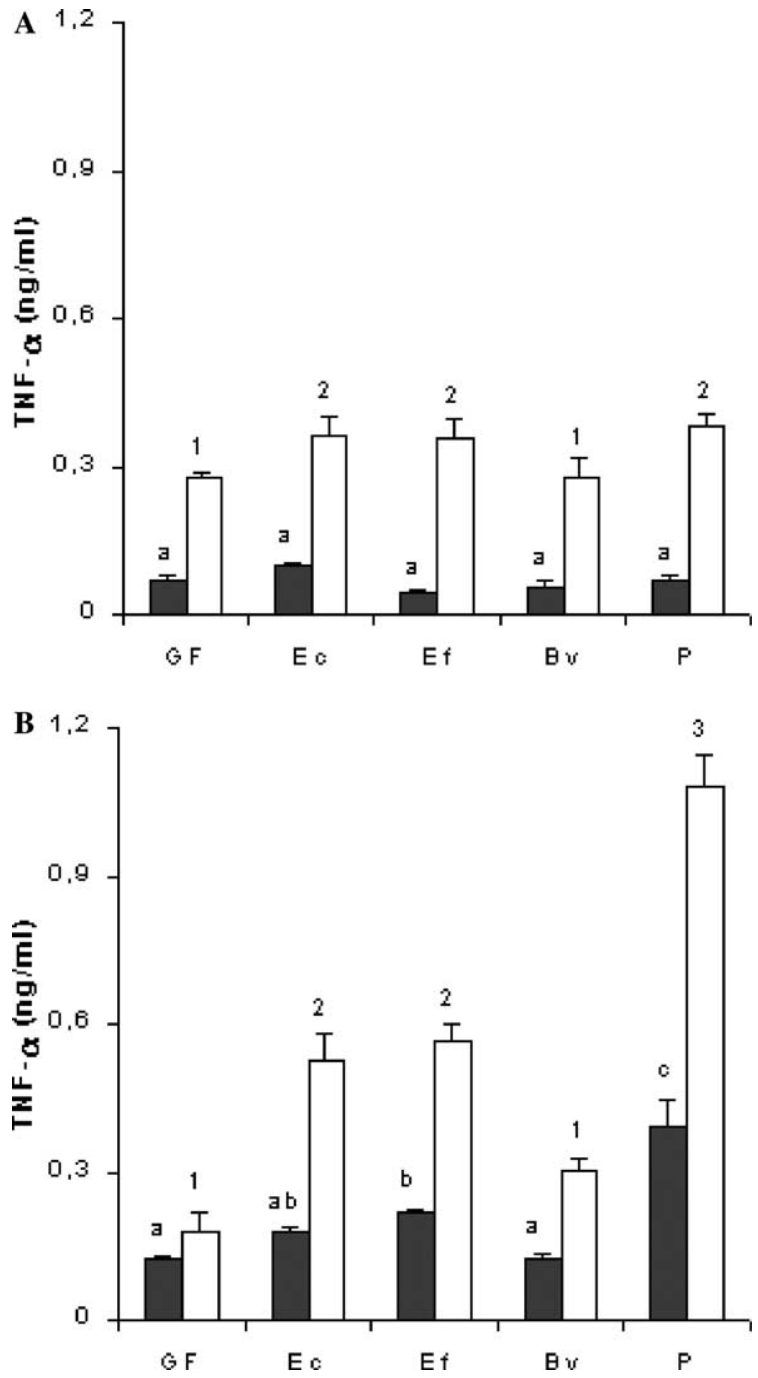

Fig. 3. TNF- $\alpha$ production by spleen cells from Swiss/NIH mice, germfree (GF) or mono-associated with Escherichia coli (EC), Enterococcus faecalis (EF), Bacteroides vulgatus (BV), and Peptostreptococcus sp. (P) before (A) and seven days after (B) intraperitoneal challenge with $5 \times 10^{3}$ trypomastigotes of Trypanosoma cruzi. Cells were cultured for $72 \mathrm{~h}$ in the absence ( $\square$ ) or presence $(\square)$ of $T$. cruzi antigen. Each bar represents the mean of one representative experiment of three performed (three mice/experiment, spleens from each mouse were cultured individually in each experiment). Vertical lines represent standard deviations of the means. ${ }^{\mathrm{a}, \mathrm{b}, \mathrm{c}}$ Different letters and ${ }^{1,2,3}$ different numbers indicate statistically significant difference between non-stimulated and stimulated groups, respectively, as evaluated by analysis of variance $(P<0.05)$.

group also had the highest infected/non-infected IFN- $\gamma$ production ratio. Germ-free mice presented the lowest calculated ratios.

\subsection{Immunoglobulin determinations}

Since the ratio of $\operatorname{IgG} 2 \mathrm{a}$ and $\mathrm{IgG} 1$ is usually an indication of the prevalence of type 1 or type 2 responses in vivo, we assayed total and T. cruzi-specific IgG subclasses in sera of mice. Higher concentrations of total 
Table 1

Nitrite production $(\mu \mathrm{M})$ in supernatants of spleen cell cultures from Swiss/NIH mice, germ-free or mono-associated with Escherichia coli, Enterococcus faecalis, Bacteroides vulgatus, and Peptostreptococcus sp. before and seven days after intraperitoneal challenge with $5 \times 10^{3}$ trypomastigotes of Trypanosoma cruzi

\begin{tabular}{|c|c|c|c|c|c|}
\hline & \multicolumn{5}{|l|}{ Association } \\
\hline & Germ-free & E. coli & E. faecalis & B. vulgatus & Peptostreptococcus \\
\hline Non-infected & $4.98 \pm 1.23^{\mathrm{a}}$ & $4.89 \pm 1.06^{\mathrm{a}}$ & $3.45 \pm 0.10^{\mathrm{a}}$ & $5.48 \pm 0.10^{\mathrm{a}}$ & $5.97 \pm 0.50^{\mathrm{a}}$ \\
\hline Infected & $5.85 \pm 0.21^{\mathrm{a}}$ & $16.51 \pm 2.63^{b}$ & $7.07 \pm 0.76^{\mathrm{a}}$ & $7.68 \pm 1.70^{\mathrm{a}}$ & $17.78 \pm 1.67^{\mathrm{b}}$ \\
\hline
\end{tabular}

a,b Different letters indicate statistically significant difference between groups for the same infected or non-infected status, as evaluated by analysis of variance $(P<0.05)$.
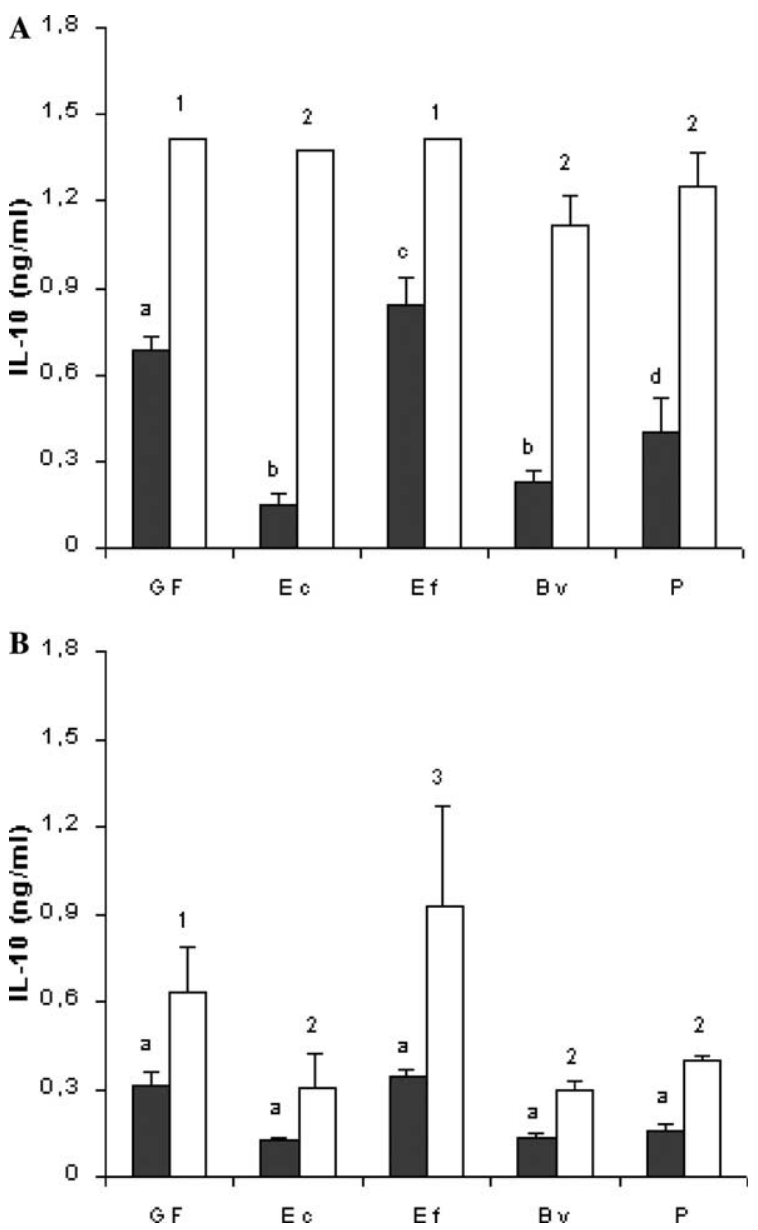

Fig. 4. IL-10 production by spleen cells from Swiss/NIH mice, germfree (GF) or mono-associated with Escherichia coli (EC), Enterococcus faecalis (EF), Bacteroides vulgatus (BV), and Peptostreptococcus $\mathrm{sp} .(\mathrm{P})$ before (A) and seven days after (B) intraperitoneal challenge with $5 \times 10^{3}$ trypomastigotes of Trypanosoma cruzi. Cells were cultured for $72 \mathrm{~h}$ in the absence $(\square)$ or presence $(\square)$ of $T$. cruzi antigen. Each bar represents the mean of one representative experiment of three performed (three mice/experiment, spleens from each mouse were cultured individually in each experiment). Vertical lines represent standard deviations of the means. ${ }^{a, b, c, d}$ Different letters and ${ }^{1,2,3}$ different numbers indicate statistically significant difference between non-stimulated and stimulated groups, respectively, as evaluated by analysis of variance $(P<0.05)$.

IgG1 were found, before the infection, in the serum of germ-free and $E$. faecalis-associated group than in the other gnotobiotic animals $(P<0.05)$. After the experi- mental challenge, the levels of this immunoglobulin decreased. Similar values were found in germ-free, $E$. coli and $E$. faecalis-associated groups, and lower concentrations $(P<0.05)$ were found for the other gnotobiotic groups (Fig. 5A). Similar concentrations of total IgG2a were observed in the serum of germ-free and gnotobiotic animals, before the infection. After the infection, levels of this immunoglobulin increased to similar values for all groups, except for the Peptostreptococcus-associated mice (Fig. 5B), which showed slightly higher levels of IgG2a.

Finally, Fig. 6 shows that both $T$. cruzi-specific IgG1 and IgG2a increased after infection. Although no statistical differences were found among groups before infection, E. faecalis mono-associated mice presented higher levels of anti-T. cruzi IgG1 and IgG2a. The other monoassociated groups showed levels of IgG1 and IgG2a similar to those found in germ-free infected animals.

\section{Discussion}

Similar to infections with other intracellular pathogens (Leishmania, Mycobacterium, and Listeria), where a strong Th1 response is protective whereas a Th2 response increases susceptibility to infection, several reports show that a Th1 response promotes protection to T. cruzi (Hoft et al., 2000; Michailowsky et al., 2001) and a Th2 response promotes susceptibility (Oliveira et al., 1996). Protection during the acute phase has been shown to be dependent on IFN- $\gamma$, and many reports describe activation of macrophages by this cytokine to produce NO and kill the obligate intracellular amastigote form of the parasite (Vespa et al., 1994). In addition, TNF- $\alpha$ provides a second signal for stimulation of NO production and anti- $T$. cruzi activity in IFN- $\gamma$ activated macrophages (Silva et al., 1995). On the other hand, the down-regulatory cytokines IL-10 and TGF- $\beta$ are associated with susceptibility to infection by inhibiting IFN- $\gamma$-mediated macrophage activation (Cardillo et al., 1996).

In the present study, germ-free and gnotobiotic mice were used to determine the influence of four predominant bacterial components of the human indigenous intestinal microbiota on the survival, production of cytokines, and immunoglobulins during the course of experimental 
Table 2

Impact of infection on cytokine production ratio

\begin{tabular}{lllll}
\hline & Association & & & \\
\cline { 2 - 5 } & Germ-free & E. coli & E. faecalis & B. vulgatus \\
\hline IFN- $\gamma / \mathrm{IL}-10^{\mathrm{a}}$ & $0.37 \pm 0.10$ & $44.28 \pm 8.09$ & $5.14 \pm 1.33$ & $6.74 \pm 0.13$ \\
IFN- $\gamma$ I/NI & $0.17 \pm 0.01$ & $9.60 \pm 1.72$ & $2.51 \pm 0.64$ & $3.60 \pm 0.62$ \\
\hline
\end{tabular}

${ }^{\text {a }}$ IFN- $\gamma /$ IL-10 in infected mice/IFN- $\gamma / \mathrm{IL}-10$ in non-infected mice.

${ }^{b}$ IFN- $\gamma$ in infected mice/IFN- $\gamma$ in non-infected mice ratio.
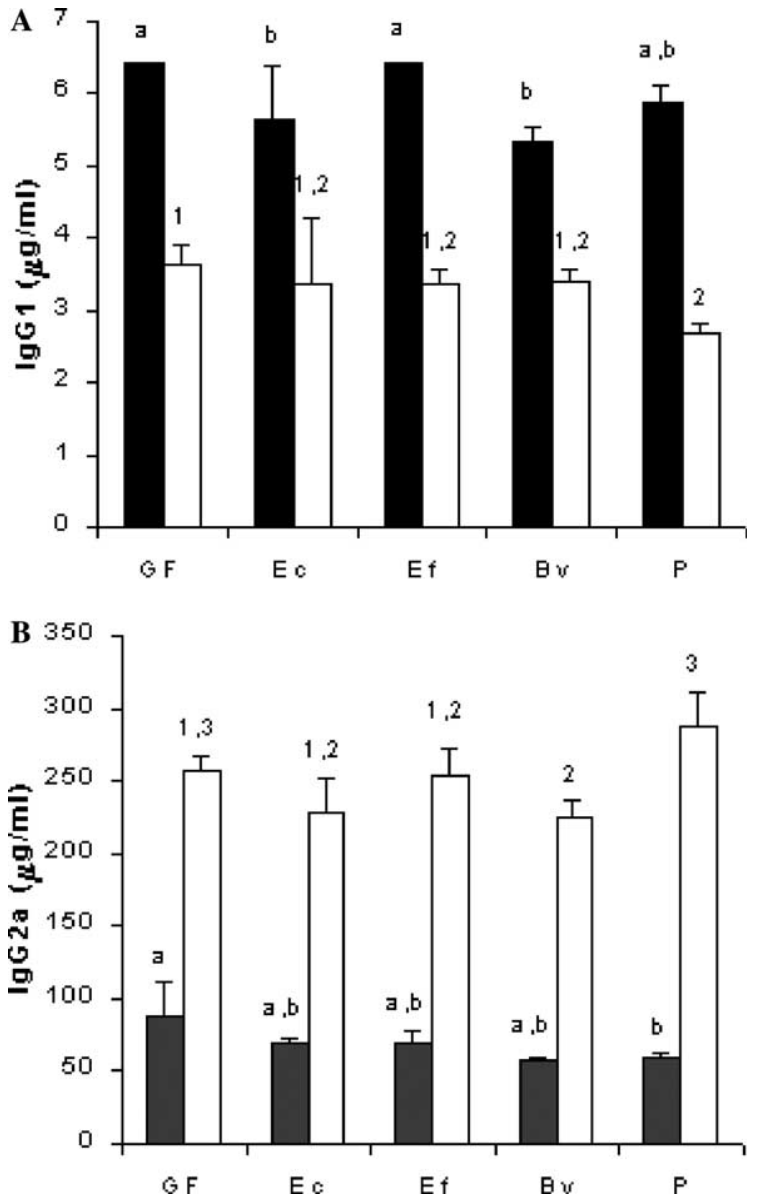

Fig. 5. Total IgG1 (A) and total IgG2a (B) in serum from Swiss/NIH mice, germ-free (GF) or mono-associated with Escherichia coli (EC), Enterococcus faecalis (EF), Bacteroides vulgatus (BV), and Peptostrep-

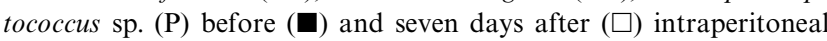
challenge with $5 \times 10^{3}$ trypomastigotes of Trypanosoma cruzi. Each bar represents the mean of one representative experiment of three performed (three mice/experiment, sera from each mouse were assayed individually). Vertical lines represent standard deviations of the means. ${ }^{a, b}$ Different letters and ${ }^{1,2,3}$ different numbers indicate statistically significant difference between non-infected and infected groups, respectively, as evaluated by analysis of variance $(P<0.05)$.

Chagas disease. A less severe T. cruzi experimental infection in conventional mice than in germ-free animals was repeatedly described in different reports published by our group along the last 15 years (Cintra et al., 1998; Furarah et al., 1991; Pedrosa et al., 1993; Santos et al., 1992; Silva et al., 1987). In the present study, a difference in survival was observed between gnotobiotic animals mono-associ-
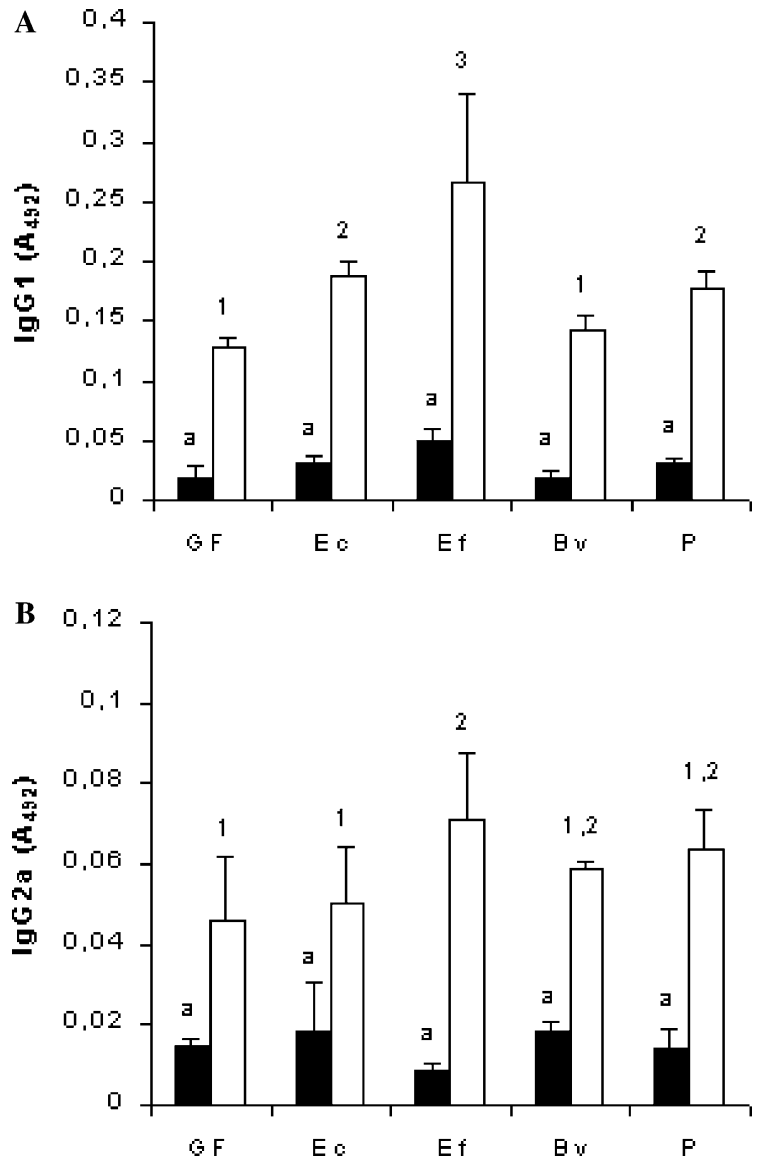

Fig. 6. Anti-Trypanosoma cruzi IgG1 (A) and anti-T. cruzi IgG2a (B) in serum from Swiss/NIH mice, germ-free (GF) or mono-associated with Escherichia coli (EC), Enterococcus faecalis (EF), Bacteroides

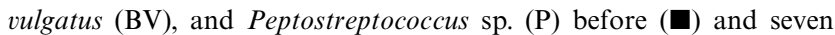
days after $(\square)$ intraperitoneal challenge with $5 \times 10^{3}$ trypomastigotes of T. cruzi. Each bar represents the mean of one representative experiment of three performed (three mice/experiment, sera from each mouse were assayed individually). Vertical lines represent standard deviations of the means. a Different letters and ${ }^{1,2,3}$ different numbers indicate statistically significant difference between non-infected and infected groups, respectively, as evaluated by analysis of variance $(P<0.05)$.

ated with E. faecalis, B. vulgatus or Peptostreptococcus sp. and germ-free mice. This protection was not so clear in $E$. coli-associated animals. Additionally, a stronger Th1 response was found in all the gnotobiotic groups after infection. Thus, higher IFN- $\gamma$, TNF- $\alpha$, and NO productions by spleen cell cultures, and higher serum levels of specific immunoglobulins of $\mathrm{IgG} 2 \mathrm{a}$ isotype were 
observed seven days after infection in gnotobiotic animals when compared to their germ-free counterparts. Basal productions of IFN- $\gamma$ by spleen cells were similar between germ-free and gnotobiotic mice before the infection, and were increased $(P<0.05)$ only in gnotobiotic animals after the infectious challenge. Spleen cells from T. cruzi-infected germ-free mice were unable to produce IFN- $\gamma$ after antigen stimulation in vitro, as opposed to gnotobiotic animals. As can be seen in Table 2, all monoassociated mice had a ratio of production of IFN- $\gamma$ after/ before infection higher than the germ-free one, particularly in $E$. coli-associated mice. Similar results were obtained for TNF- $\alpha$ production after infection. In accordance with the higher IFN- $\gamma$ and TNF- $\alpha$ levels in spleen cell cultures from gnotobiotic mice infected with $T$. cruzi, an increase in NO production was also noted in these animals, except for E. faecalis-associated mice. Only, cells from germ-free and E. faecalis-associated infected group produced IL-10 after in vitro stimulation with antigen. However, before infection, IL-10 was produced after in vitro stimulation by cells from all groups. No IL-4 was detected in cell cultures from any of the groups. Hence, a higher production of type 1 cytokines was found in cultures from infected gnotobiotic mice, but with some difference among mono-associated groups. This can be seen in Table 2, which shows that all mono-associated mice displayed a high IFN- $\gamma / \mathrm{IL}-10$ ratio, indicating a strong pro-inflammatory response with a more pronounced phenomenon observed in E. coli-associated animals.

In accordance to the cytokine data, infected gnotobiotic mice showed slightly higher levels of $T$. cruzi-specific $\mathrm{IgG} 2 \mathrm{a}$ in sera, after infection. These results are in agreement with the classical work by MacDonald and Carter (1979) who showed that germ-free mice had a deficiency in mounting a cell-mediated immune response, compared to conventional controls. Interestingly, we also detected an increase in the levels of $T$. cruzi-specific IgG1 after infection. Although this observation may, in principle, contradict the Th1/Th2 profile, it has been shown that IgG1 production can be observed in IL-4-deprived mice and thus, would not be associated with a Th2 type response. On the contrary, this non-anaphylactic IgG1 would be induced by IL-12 (Faquim-Mauro et al., 1999). Nevertheless the measurements of total IgG levels in our study reflect a shift towards a Th1 response (Fig. 5A).

IFN- $\gamma$ production by spleen cells from uninfected germ-free, B. vulgatus, E. faecalis-, and Peptostreptococcus-associated mice in response to $T$. cruzi antigen was higher than in the E. coli-associated group. This unpredicted observation might be explained by the lower stimulation by LPS in the germ-free and Gram positive associated mice, since exposure to low levels of LPS has been shown to decrease, at least partially, IFN- $\gamma$ production to further LPS stimuli (Erroi et al., 1993; Henricson et al., 1990). According to Ropert et al. (2002), LPS can induce tolerance to tGPI mucins and vice-versa (Ropert et al., 2002). Hence, it is possible that E. coli-associated mice are exposed to low levels of LPS from the enterobacterium and were, therefore, less reactive to $T$. cruzi antigens (which contain tGPI mucins). Heterogeneity and difference in LPS immunogenic potential between Gram negative bacteria are well known (Ogawa et al., 1997 ) and these differences could explain why B. vulgatus, another Gram negative bacterium, did not induce tolerance to tGPI mucins.

Due to the higher production of protective cytokines by gnotobiotic animals in response to infection, protection as measured by mortality was found in our experiments. This is not surprising, since the presence of these pro-inflammatory cytokines has been extensively associated with resistance to T. cruzi (Hoft et al., 2000; Michailowsky et al., 2001). However, E. coli mono-associated mice were more susceptible to $T$. cruzi than the other mono-associated animals. Interestingly, cells from noninfected $E$. coli mono-associated mice produced less IFN- $\gamma$ upon antigenic stimulation than the other groups (Fig. 2A), suggesting a decreased innate response to this stimulus in these animals that could be related either to a decreased IL-12 production or to a lack of NK cell activity. This decreased response would impair the early control of the parasite resulting in lower survival. We hypothesize that, due to the lack of background IFN- $\gamma$ production to $T$. cruzi antigens found before infection in these mice, early protective response to the parasite was impaired, hence the lower survival.

It is possible that priming of $\mathrm{T}$ cells by the indigenous microbiota influences the memory cell repertoire in conventional mice, hence influencing their immune response. Indeed, an exacerbated early IL-4 production in response to infection with Leishmania major has been associated with a $\mathrm{CD} 4^{+} \mathrm{T}$ cell population expressing the memory phenotype (possibly primed by E. coli) in BALB/c mice (Julia et al., 2000). However, more recent data have associated the early IL-4 production in response to the parasite to a naïve phenotype (Stetson et al., 2002), probably ruling out the role of the indigenous microbiota in skewing the immune response during infection with $L$. major. The number of T cells expressing the memory phenotype in germ-free mice is also controversial: some authors find that there are more cells expressing memory markers when mice are exposed to the indigenous microbiota (Inagaki et al., 1996; Lee et al., 1990; Price and Cerny, 1999), others do not (Bonorino et al., 1998; Dobber et al., 1992; Park et al., 2000). Hence, the extent to which the indigenous microbiota can change the host $\mathrm{T}$ cell repertoire and influence its response to pathogens is still not clear.

Another possible influence that the microbiota may have is in the regulatory cell repertoire. Several studies have suggested that low exposition to microorganisms would induce an exacerbated immune response, either of 
the Th1 or of the Th2 type (Wills-Karp et al., 2001; Yazdanbakhsh et al., 2002). However, our data show that the lack of exposition to antigenic stimuli from the indigenous microbiota does not induce an exacerbated immune response against a single invasive microorganism, suggesting that there might be a threshold of antigenic stimulation below which an inflammatory response is not fully mounted.

In conclusion, this study provides evidence that components of the indigenous microbiota play an important role in the development of an immune system that is competent to react against an acute infection. However, the experiments with gnotoxenic animals mono-associated with bacterial strains representative of the predominant gut microbiota of human showed that some strains may be more effective than others in interfering in the Th1-Th2 balance.

\section{Acknowledgments}

This study was supported by grants and fellowships from the Conselho Nacional do Desenvolvimento Científico e Tecnológico (CNPq) and Fundação de Amparo à Pesquisa do Estado de Minas Gerais (FAPEMIG). The authors acknowledge Dr. Olindo Assis Martins Filho (Centro de Pesquisa René Rachou, FIOCRUZ, Belo Horizonte, MG, Brazil) for very helpful suggestions. The authors are grateful to Maria Gorete Barbosa Ribas for valuable technical help, and to Ronilda Maria de Paula (in memoriam), Maria Helena Alves de Oliveira and Antônio Mesquita Vaz for animal care.

\section{References}

Berg, R.D., 1996. The indigenous gastrointestinal microflora. Trends in Microbiology 4, 430-435.

Bonorino, C., Nardi, N.B., Zhang, X., Wysocki, L.J., 1998. Characteristics of the strong antibody response to mycobacterial Hsp70: a primary, $\mathrm{T}$ cell-dependent $\mathrm{IgG}$ response with no evidence of natural priming or gamma delta $\mathrm{T}$ cell involvement. Journal of Immunology 161, 5210-5216.

Brener, Z., 1962. Therapeutic activity and criterion of cure on mice experimentally infected with Trypanosoma cruzi. Revista do Instituto de Medicina Tropical de São Paulo 4, 389-396.

Cardillo, F., Voltarelli, J.C., Reed, S.G., Silva, J.S., 1996. Regulation of Trypanosoma cruzi infection in mice by IFN- $\gamma$ and IL-10: the role of NK cells. Infection and Immunity 64, 128-134.

Cintra, I.P., Silva, M.E., Silva, M.E., Silva, M.E.C., Crocco-Afonso, L.C., Nicoli, J.R., Bambirra, E.A., Vieira, E.C., 1998. Influence of dietary protein content on Trypanosoma cruzi infection in germfree and conventional mice. Revista do Instituto de Medicina Tropical de São Paulo 40, 355-362.

Dobber, R., Hertogh-Huijbregts, A., Rozing, J., Bottomly, K., Nagelkerken, L., 1992. The involvement of the intestinal microflora in the expansion of $\mathrm{CD}^{+} \mathrm{T}$ cells with a naive phenotype in the periphery. Development Immunology 2, 141-150.

Duarte, R., Silva, A.M., Vieira, L.Q., Afonso, L.C.C., Nicoli, J.R., 2004. Influence of normal microbiota on some aspects of the immune response during experimental infection with Trypanosoma cruzi in mice. Journal of Medical Microbiology 53, 741-748.

Erroi, A., Fantuzzi, G., Mengozzi, M., Sironi, M., Orencole, S.F., Clark, B.D., Dinarello, C.A, Isetta, A., Gnocchi, P., Giovarelli, M., 1993 Differential regulation of cytokine production in lipopolysaccharide tolerance in mice. Infection and Immunity 61, 4356-4359.

Faquim-Mauro, E.L., Coffman, R.L., Abrahamsohn, I.A., Macedo, M.S., 1999. Mouse IgG1 antibodies comprise two functionally distinct types that are differentially regulated by IL-4 and IL-12. Journal of Immunology 163, 3572-3576.

Furarah, A.M., Crocco-Afonso, L.C., Silva, M.E.C., Silva, M.E., Silva, M.E., Bambirra, E.A., Vieira, E.C., Nicoli, J.R., 1991. Immune response of germ-free mice to experimental infection with Trypanosoma cruzi. Brazilian Journal of Medical and Biological Research 24, 1223-1231.

Gouet, P., Yvore, P., Naciri, M., Contrepois, M., 1984. Influence of digestive flora on parasite development and the pathogenic effect of Eimeria ovinoidalis in the axenic, gnotoxenic and conventional lamb. Research in Veterinary Sciences 36, 21-23.

Green, L., Wagner, D., Glogowski, J., Skipper, P., Wishnok, J., Tannenbaum, S., 1982. Analysis of nitrate, nitrite and $(15 \mathrm{~N})$ nitrate in biological fluids. Analytical Biochemistry 126, 131-138.

Harleman, J.H., Meyer, R.C., 1984. Life cycle of Isopora suis in gnotobiotic and conventional piglets. Veterinary Parasitology 17, 27-39.

Henricson, B.E., Benjamin, W.R., Vogel, S.N., 1990. Differential cytokine induction by doses of lipopolysaccharide and monophosphoryl lipid $\mathrm{A}$ that result in equivalent early endotoxin tolerance. Infection and Immunity 58, 2429-2437.

Hoft, D.F., Schnap, A.R., Eickhoff, C.S., Roodman, S.T., 2000. Involvement of $\mathrm{CD}^{+}{ }^{+} \mathrm{Th} 1$ cells in systemic immunity protective against primary and secondary challenges with Trypanosoma cruzi. Infection and Immunity 68, 197-204.

Inagaki, H., Suzuki, T., Nomoto, K., Yoshikai, Y., 1996. Increased susceptibility to primary infection with Listeria monocytogenes in germfree mice may be due to lack of accumulation of $\mathrm{L}$-selectin $+\mathrm{CD} 44^{+} \mathrm{T}$ cells in sites of inflammation. Infection and Immunity 64, 3280-3287.

Johnson, J., Reid, W.M., 1973. Ascaridia galli (Nematoda): development and survival in gnotobiotic chickens. Experimental Parasitology $33,95-99$.

Julia, V., McSorley, S.S., Malherbe, L., Breittmayer, J.P., Girard-Pipau, F., Beck, A., Glaichenhaus, N., 2000. Priming by microbial antigens from the intestinal flora determines the ability of $\mathrm{CD}^{+} \mathrm{T}$ cells to rapidly secrete IL-4 in BALB/c mice infected with Leishmania major. Journal of Immunology 165, 5637-5645.

Lee, W.T., Yin, X.M., Vitetta, E.S., 1990. Functional and ontogenetic analysis of murine CD45Rhi and CD45Rlo $\mathrm{CD}^{+} \mathrm{T}$ cells. Journal of Immunology 144, 3288-3295.

MacDonald, T.T., Carter, P.B., 1979. Requirement for a bacterial flora before mice generate cells capable of mediating the delayed hypersensitivity reaction to sheep red blood cells. Journal of Immunology $122,2624-2629$.

MacFarland, L.V., 2000. Normal flora: diversity and functions. Microbial Ecology in Health and Disease 12, 193-207.

Martins, W.A., Melo, A.L., Nicoli, J.R., Cara, D.C., Carvalho, M.A.R., Lana, M.A., Vieira, E.C., Farias, L.M., 2000. A method of decontaminating Strongyloides venezuelensis larvae for the study of strongyloidiasis in germfree and conventional mice. Journal of Medical Microbiology 49, 387-390.

Michailowsky, V., Silva, N.M., Rocha, C.D., Vieira, L.Q., Lannes-Vieira, J., Gazzinelli, R.T., 2001. Pivotal role of interleukin-12 and interferon- $\gamma$ axis in controlling tissue parasitism and inflammation in the heart and central nervous system during Trypanosoma cruzi infection. American Journal of Pathology 159, 1723-1733.

National Research Council, 1996. Guide for the Care and Use of Laboratory Animals. National Academy Press, Washington.

Ogawa, T., Suda, Y., Kashihara, W., 1997. Immunobiological activities of chemically defined lipid A from Helicobacter pylori lipopolysac- 
charide in comparison with Porphyromonas gingivalis lipid A and Escherichia coli-type synthetic lipid A (compound 506). Vaccine 15, 1598-1605.

Oliveira, L.C.B., Lafaille, M.A.C., Lima, G.M.C.A., Abrahamsohn, I.A., 1996. Antigen-specific IL-4- and IL-10-secreting CD4 ${ }^{+}$lymphocytes increase in vivo susceptibility to Trypanosoma cruzi infection. Cell Immunology 170, 41.

Owen, D., 1975. Eimeria falciformis (Eimer, 1870) in specific pathogen free and gnotobiotic mice. Parasitology 71, 293-303.

Park, S.H., Benlagha, K., Lee, D., Balish, E., Bendelac, A., 2000. Unaltered phenotype, tissue distribution and function of Valpha14(+) NKT cells in germ-free mice. European Journal of Immunology 30, 620-625.

Pedrosa, M.L., Nicoli, J.R., Silva, M.E., Silva, M.E., Silva, M.E.C., Vieira, L.Q., Bambirra, E.A., Vieira, E.C., 1993. The effect of iron nutritional status on Trypanosoma cruzi infection in germ-free and conventional mice. Comparative Biochemistry and Physiology A 106, 813-821.

Phillips, B.P., Wolfe, P.A., 1959. The use of germ-free guinea pigs in studies on the microbial interrelationships in amoebiasis. Annals of the New York Academy of Sciences 78, 308-314.

Price, P.W., Cerny, J., 1999. Characterization of CD4 ${ }^{+} \mathrm{T}$ cells in mouse bone marrow. I. Increased activated/memory phenotype and altered TCR Vbeta repertoire. European Journal of Immunology 29, 1051-1056.

Przyjalkowski, Z., Wescott, R.B., 1969. Trichinella spiralis: establishment in gnotobiotic mice affected by Bacillus mesentericus, Bacillus subtilis and Pseudomonas aeruginosa. Experimental Parasitology $25,8-12$.

Reid, W.M., Botero, H., 1967. Growth of the cestode Raillietina cesticillus in bacteria-free chickens. Experimental Parasitology 21, 149-153.

Ropert, C., Ferreira, L.R., Campos, M.A., Procópio, D.O., Travassos, L.R., Ferguson, M.A., Reis, L.F., Teixeira, M.M., Almeida, I.C., Gazzinelli, R.T., 2002. Macrophage signaling by glycosylphosphatidylinositol-anchored mucin-like glycoproteins derived from Trypanosoma cruzi trypomastigotes. Microbes and Infection 4, 10151025.

Rutter, J.M., Beer, R.J.S., 1975. Synergism between Trichuris suis and the microbial flora of the large intestine causing dysentery in pigs. Infection and Immunity 11, 395-404.

Salkowski, C.A., Bartizal, K.F., Balish, M.J., Balish, E., 1987. Colonization and pathogenesis of Cryptococcus neoformans in gnotobiotic mice. Infection and Immunity 55, 2000-2005.
Santos, C.F., Silva, M.E., Silva, M.E., Silva, M.E.C., Nicoli, J.R., Crocco-Afonso, L.C., Santos, J.E., Bambirra, E.A., Vieira, E.C., 1992. Effect of essential fatty acid deficient diet on experimental infection with Trypanosoma cruzi in germ-free and conventional mice. Brazilian Journal of Medical and Biological Research 25, 795-803.

Silva, M.E., Evangelista, E.A., Nicoli, J.R., Bambirra, E.A., Vieira, E.C., 1987. American trypanosomiasis (Chagas disease) in conventional and germ-free rats and mice. Revista do Instituto de Medicina Tropical de São Paulo 29, 284-288.

Silva, J.S., Vespa, G.N.R., Cardoso, M.A.G., Aliberti, J.C., Cunha, F.Q., 1995. Tumor necrosis factor alpha mediates resistance to Trypanosoma cruzi in mice by inducing nitric oxide production in infected IFN- $\gamma$-activated macrophages. Infection and Immunity 63, 48624867.

Stetson, D.B., Mohrs, M., Mallet-Designe, V., Teyton, L., Locksley, R.M., 2002. Rapid expansion and IL-4 expression by Leishmaniaspecific naive helper T cells in vivo. Immunity 17, 191-200.

Torres, M.F., Uetanabaro, A.P.T., Costa, A.F., Alves, C.A., Farias, L.M., Bambirra, E.A., Penna, F.J., Vieira, E.C., Nicoli, J.R., 2000. Influence of bacteria from the duodenal microbiota of patients with symptomatic giardiasis on the pathogenicity of Giardia duodenalis in gnotoxenic mice. Journal of Medical Microbiology 49, 209-215.

Vespa, G.N.R., Cunha, F.Q., Silva, J.S., 1994. Nitric oxide is involved in the control of Trypanosoma cruzi induced parasitemia and directly kills parasite in vitro. Infection and Immunity 62, 5177-5182.

Visco, R.J., Barnes, W.C., 1972. Eimeria tenella in bacteria-free and conventionalized chicks. Journal of Parasitology 58, 323-331.

Wescott, R.B., 1968. Experimental Nematospiroides dubius infection in germ-free and conventional mice. Experimental Parasitology 22, 245-249.

Wescott, R.B., Todd, A.C., 1964. A comparison of the development of Nippostrongylus brasiliensis in germ-free and conventional mice. Journal of Parasitology 50, 138-143.

Wills-Karp, M., Santeliz, J., Karp, C.L., 2001. The germless theory of allergic disease revisiting the hygiene hypothesis. Nature Review of Immunology 1, 69-75.

Wilson, K.H., 1995. Ecological concepts in the control of pathogens. In: Roth, J.A. (Ed.), Virulence Mechanisms of Bacterial Pathogens. American Society for Microbiology, Washington, pp. 245-256.

Yazdanbakhsh, M., Kremsner, P.G., van Ree, R., 2002. Allergy, parasites, and the hygiene hypothesis. Science 296, 490-494. 